Obere Extremität 2020 $15: 333$

C) Springer Medizin Verlag $\mathrm{GmbH}$, ein Teil von https://doi.org/10.1007/s11678-020-00610-z

Online publiziert: 22 . Oktober 2020

Springer Nature 2020

\section{Franziska Haas}

Deutsches Gelenkzentrum, ATOS Klinik Heidelberg, Heidelberg, Deutschland

\section{Buchbesprechung}

nunmehr seinem Anspruch gerecht, eine allumfassende und insbesondere schulunabhängige Darstellung der Techniken der Manuellen Medizin im gesamten deutschsprachigen Raum abzubilden. Auf 584 Seiten und mit Hilfe von 1660 Abbildungen und Fotografien erfüllt es dieses ambitionierte Vorhaben zweifellos.

Insbesondere die in Band 1 der Reihe gesondert abgehandelten theoretischen Grundlagen der Manuellen Medizin sowie deren klinische Integration und Anwendung geben dem vorliegenden Werk den Raum, die praktische Anwendung diagnostischer und therapeutischer Techniken in Form eines ganzheitlichen Nachschlagewerks ausführlich darzulegen. Eine übersichtliche Zusammenfassung jener Grundlagen sowie ein Symbolverzeichnis und Glossar bilden das abschließende Kapitel des zweiten Bands und ermöglichen das rasche Nachschlagen.

Bei der Betrachtung der Neuauflage fällt insbesondere die Möglichkeit der digitalen Nutzung ins Auge. Der beiliegende Zugangscode zur Online-Plattform eRef des Georg Thieme Verlags erlaubt die überaus praktische mobile Nutzung. Insbesondere hervorzuheben ist, dass durch das EPUB-Format („electronic publication") die automatische dynamische Anpassung des Textes an die Größe aller mobilen Anzeigegeräte erfolgt. Somit besteht die Möglichkeit, auch ein solch allumfassendes Werk jederzeit mitzuführen, sei es auf dem Smartphone, Tablet oder E-BookReader.

Zum systematischen Erlernen und Nachschlagen der Techniken der Manuellen Medizin soll jedoch die gebundene Druckversion keinesfalls geschmälert sein. In acht ausführlichen Kapiteln werden alle relevanten Techniken der diagnostischen und therapeutischen Manuellen Medizin von kranial nach kaudal aufgeführt. Die ausführlichen Erklärungen werden durch Fotos und schemati- sche Abbildungen aus dem Anatomieatlas der „Prometheus“-Reihe des Georg Thieme Verlags hervorragend ergänzt.

Insbesondere bei der Darstellung komplexer Sachverhalte trägt die schrittweise schematische Darstellung enorm zur Übersichtlichkeit bei und bietet dem Leser einen sicher nachvollziehbaren Handlungsleitfaden. Hier sei beispielsweise die diagnostische Untersuchung des Schultergelenks mit seinen ausgeprägten Freiheitsgraden und einer Vielzahl sich teils bedingender Funktionen und damit einhergehend auch Pathologien aufgeführt. Dank der eingefügten Symbolik ermöglichen die Fotografien die Verbildlichung statischer wie auch dynamischer Prozesse in gleichsam verständlicher Weise.

Ergänzend werden in vielen Kapiteln jeweils die Schmerzausstrahlungsmuster („referred pain patterns") und Techniken der myofaszialen Triggerpunkttherapie des jeweiligen Bewegungssystems aufgeführt. Außerdem sind zahlreiche Vorschläge für Heimübungen zur selbstständigen Fortführung der eingeleiteten Behandlung angeboten. Somit ist ein umfassender Leitfaden gegeben, den $\mathrm{Pa}$ tienten ganzheitlich $\mathrm{zu}$ behandeln und bezüglich der ergänzenden Therapie in Eigenregie zu beraten.

„Manuelle Medizin 2 - Diagnostische und therapeutische Techniken praktisch anwenden" bietet praktisch tätigen Kollegen der Fachgebiete der muskuloskelettalen Medizin mit der übersichtlich gegliederten ausführlichen Darstellung ein ganzheitliches Lehr- und Nachschlagewerk, welches sich dank der ausgiebigen, teils dynamischen Illustrierung lebendig darstellt und ein gutes Preis-LeistungsVerhältnis bietet.

Franziska Haas (Heidelberg) (SAMM) wurde anlässlich der Überarbeitung durch Weiterbildungsinhalte des Ärzteseminars MWE Isny Neutrauchburg ergänzt. Dadurch wird das Gesamtwerk 